\title{
CrystEngComm
}

Check for updates

Cite this: CrystEngComm, 2019, 21 1293

Received 17th October 2018,

Accepted 12th January 2019

DOI: $10.1039 / c 8 c e 01778 f$

rsc.li/crystengcomm

\section{Synthesis of size-tuneable $\beta$-FeOOH nanoellipsoids and a study of their morphological and compositional changes by reduction $\uparrow$}

\author{
Georgios Kasparis, (iD) ab Aritz Sterne Erdocio, ${ }^{\text {ab }}$ \\ Joshua Mark Tuffnell ${ }^{b}$ and Nguyen Thi Kim Thanh iD *ab
}

\begin{abstract}
Shape anisotropy has been shown to positively influence the performance of nanoparticles in many applications. Size-tuneable akaganéite $(\beta-\mathrm{FeOOH})$ nanoellipsoids have been prepared and used as precursors for producing anisotropic iron oxide nanoparticles by reduction. Nanoellipsoids with a size between 25$235 \mathrm{~nm}$ in length and aspect ratio of 5 or 6, compatible for biomedical and catalytic applications, were obtained by using different amounts and molecular weights of branched polyethyleneimine. The reaction was scaled-up by a factor of two leading to a yield increase of $55 \%$. Oleylamine or hydrazine was used to reduce $\beta$-FeOOH nanoellipsoids at different reaction temperatures and times. With oleylamine at $200{ }^{\circ} \mathrm{C}$, the same nanoellipsoid shape was retained with clear voids, but the phase changed to oxide-hydroxide; while at a higher temperature of $250^{\circ} \mathrm{C}$, the phase converted to iron oxide but the nanoparticles lost their original morphology. In the case of hydrazine, with the presence of polyethyleneimine, goethite $(\alpha-\mathrm{FeOOH})$ wires were formed, the amount of which is proportionally increased with time and concentration of the polymer; without the latter, iron oxide particles were formed of variable shapes.
\end{abstract}

\section{Introduction}

Nanomaterials have many potential applications from catalysis $^{1,2}$ to energy conversion, ${ }^{3,4}$ medicines $^{5-7}$ and environmen$\mathrm{tal}^{8,9}$ and even in military uses. ${ }^{10}$ Of great interest are anisotropic crystals such as cubes, wires, triangles, platelets and others which exhibit altered properties compared to their isotropic counterparts. Elongated nanostructures are of intense scientific interest ${ }^{11,12}$ and many materials have been shown to exhibit better performance compared to other shapes. For example, gold nanorods exhibit higher sizenormalised optical cross-section compared to gold nanospheres, ${ }^{13}$ and polymeric filomicelles had prolonged blood circulation and enhanced retention at the tumour site compared to their spherical counterparts. ${ }^{14}$ Similarly, polystyrene nanorods showed reduced non-specific attachment and enhanced accumulation to the lungs and brain, ${ }^{15}$ while silver nanorods with different aspect ratios could scatter light from visible to the near-infrared region ${ }^{16}$ in comparison with spherical nanoparticles of each material. Recently, iron oxide

\footnotetext{
${ }^{a}$ Department of Physics and Astronomy, University College London, Gower street, London WC1E 6BT, UK. E-mail: ntk.thanh@ucl.ac.uk

${ }^{b}$ UCL Healthcare Biomagnetic and Nanomaterials Laboratories, 21 Albemarle street, London W1S 4BS, UK

$\dagger$ Electronic supplementary information (ESI) available. See DOI: 10.1039/ c8ce01778f
}

nanorods were shown to induce a stronger magnetic field around themselves and therefore exhibit better contrast in Magnetic Resonance Imaging (MRI) ${ }^{17}$ and enhanced performance in magnetic hyperthermia ${ }^{18}$ compared to nanospheres. Among magnetic nanoparticles, spheres are by far the most common and facile shape to obtain and synthetic procedures of more complex shapes such as rods are scarce with only a handful of reports in the literature due to the difficulty in producing anisotropic magnetite $\left(\mathrm{Fe}_{3} \mathrm{O}_{4}\right)$ nanoparticles due to their cubic crystal habit. ${ }^{17,19,20}$ Broadly, the two approaches taken so far are to either form the iron oxide nanorods directly from an iron pentacarbonyl precursor or form nanorods of an $\mathrm{FeOOH}$ polymorph and then reduce the compound to form the iron oxide phase. The one-step syntheses of the iron oxide nanorods reported in the literature are either using bespoke reagents or suffer from very poor magnetic properties. ${ }^{19}$ The difficulty in forming elongated iron oxide structures directly is related to their cubic crystal structure with no preferential orientation for growth. To that end, different surfactants are used to arrest the growth of some crystallographic planes and allow others to grow resulting in anisotropic iron oxide nanoparticles. In contrast, $\mathrm{FeOOH}$ polymorphs often crystallise in the form of elongated structures even in the absence of any surfactants. ${ }^{21-23}$ A two-step facile synthesis produces $\beta$-FeOOH first by simple hydrolysis and then a reduction procedure converts it to the oxide. Using the two-step synthesis, some results have shown that the morphology of 
the nanorods is not preserved after reduction due to the changes in the crystal structure and efforts are made towards controlling the morphology during reduction. ${ }^{23}$ In this article, we expand on the synthesis of $\beta-\mathrm{FeOOH}$ nanoellipsoids to provide a wider range of sizes as precursors for reduction reactions to produce anisotropic iron oxide nanoparticles.

A high-temperature method has been previously tried to convert one material to another. Usually, this method causes aggregation and sintering losing the nanostructure characteristics. ${ }^{24}$ Attempts to prevent sintering and aggregation involve the use of various solvents and surfactants. Phase transformation from $\beta$-FeOOH rods to iron oxide is achieved while retaining their shape, but they have hollow structure, and weak magnetic properties. ${ }^{25,26}$

In this work, we explored further the synthesis of $\beta$-FeOOH nanoellipsoids to produce a wider range of sizes and increase the yield of the reaction by scaling-up. The reduction of the $\beta$-FeOOH nanoellipsoids with reducing agents of different reduction potentials was also investigated. We found that certain experimental parameters are important for driving the reactions to have polymorphic transformation of $\beta$-FeOOH to $\alpha-\mathrm{FeOOH}$ or reduction to iron oxide. A loss of the ellipsoidal morphology is observed when iron oxide is formed. We were able to preserve the elongated morphology in the polymorphic transformation process to produce $\alpha$-FeOOH nanowires in the presence of polyethyleneimine (PEI).

\section{Experimental}

\section{Chemicals}

Iron(III) chloride hexahydrate (99\%), branched PEI solution 50 weight (wt)\% ( $\left.M_{\mathrm{w}} 750 \mathrm{kDa}\right)$ and branched PEI $\left(M_{\mathrm{w}} 25 \mathrm{kDa}\right)$ were purchased from Merck UK. Iron(II) chloride tetrahydrate (99\%) was purchased from Honeywell, UK. Oleylamine (80$90 \%)$ and $\mathrm{HCl}(37 \%)$ were purchased from Acros Organics UK. Acetone (technical grade) was purchased from VWR, UK. Ethanol (100\%) was purchased from HaymanKimia Ltd. Water was purified by Purelab Ultra, Elga, UK.

\section{Characterisation methods}

The nanoparticles were visualised by transmission electron microscopy (TEM) using a JEOL JEM 1200-EX operating at an accelerating voltage of $120 \mathrm{kV}$ using Gatan DigitalMicrograph software. The samples were cast on carbon coated copper grids and air-dried. Size analysis was performed using image analysis software ImageJ. The X-ray diffraction (XRD) patterns of the powder samples were recorded at room temperature between $20-110^{\circ}$ on a PANalytical $X^{\prime}$ Pert ${ }^{3}$ equipped with a cobalt source $(\lambda=1.789 \AA)$ in transmission-reflection spinning mode. Thermogravimetric analysis (TGA) was performed using a Discovery TGA (TA Instruments) under a nitrogen atmosphere between room temperature up to $700{ }^{\circ} \mathrm{C}$ with a heating rate of $10{ }^{\circ} \mathrm{C} \mathrm{min}^{-1}$. The infrared spectra of the dry powder samples were recorded on a PerkinElmer Spectrum 100 attenuated total reflectance Fourier transform infrared spectrometer (ATR-FTIR) at room temperature accumulating 20 scans between $600-4000 \mathrm{~cm}^{-1}$ for each sample which were analysed with the software Spectrum. Magnetic measurements were recorded on a Quantum Design MPMS 3 superconducting quantum interference-vibrating sample magnetometer (SQUID-VSM) between \pm 70 kOe at $300 \mathrm{~K}$.

\section{Synthesis of the $\beta$-FeOOH nanoellipsoids}

A general synthesis of the $\beta$-FeOOH nanoellipsoids was previously described. ${ }^{17}$ Briefly, $\mathrm{FeCl}_{3} \cdot 6 \mathrm{H}_{2} \mathrm{O}(5.4 \mathrm{~g}, 20 \mathrm{mmol})$ was added in water $(100 \mathrm{ml})$ containing PEI. The mixture was heated at $80{ }^{\circ} \mathrm{C}$ in an oil bath for $2 \mathrm{~h}$ under magnetic stirring (500 rpm). The nanoparticles were isolated by centrifugation $(8000 \mathrm{~g}, 10 \mathrm{~min})$, rinsed with acetone and ethanol sequentially and dried. The amount of PEI, its molecular weight and the reaction time have been varied to investigate the role of each parameter in the formation of the $\beta-\mathrm{FeOOH}$ nanoellipsoids.

\section{Reduction with oleylamine}

Oleylamine was used as a mild reducing agent. $200 \mathrm{mg}$ of $\beta$-FeOOH nanoellipsoids were mixed with oleylamine $(3.14 \mathrm{~g}$, $11.7 \mathrm{mmol}$ ) in a three-neck round bottom flask. Under a nitrogen atmosphere, the mixture was heated to $200{ }^{\circ} \mathrm{C}$ under magnetic stirring $(500 \mathrm{rpm})$ for $4 \mathrm{~h}$. The product was left to cool down, washed three times with ethanol and centrifuged $(8000 \mathrm{~g}, 5 \mathrm{~min})$. The solid product was recovered from acetone and dried at $80{ }^{\circ} \mathrm{C}$ in an oven.

\section{Reduction with hydrazine}

Hydrazine was employed as a strong reducing agent. $90 \mathrm{mg}$ of $\beta$-FeOOH nanoellipsoids were dispersed in water $(20 \mathrm{ml})$ in a three-neck round bottom flask. The nanoparticles were completely resuspended after $20 \mathrm{~min}$ of magnetic stirring. Approximately $1 \mathrm{ml}$ of hydrazine monohydrate was added to reach a $\mathrm{pH}$ value of 10.5 which was determined using a Thermo Scientific Orion 3-Star pH meter. The mixture was refluxed at $100{ }^{\circ} \mathrm{C}$ under magnetic stirring (500 rpm) for $4 \mathrm{~h}$. The reaction mixture was left to cool down and the product was washed with ethanol and acetone and centrifuged $(8000 \mathrm{~g}, 5 \mathrm{~min})$ three times. The nanoparticles were dried at $80^{\circ} \mathrm{C}$ in an oven.

\section{Results and discussion}

The amount of the $\beta$-FeOOH nanoellipsoids obtained with a typical reaction was very low, limiting the characterisation and future use of the product. To achieve adequate characterisation and expedite the development of $\beta-\mathrm{FeOOH}$ as potential precursors for magnetic nanoparticle synthesis, the scalability of the reaction was initially assessed. For the scaled-up syntheses, two PEI with different molecular weights (25 and $750 \mathrm{kDa}$ ) were separately used and the amount of all the reagents was increased by a factor of two. Both reactions were successfully scaled-up as it was confirmed from the TEM images shown in Fig. 1 producing morphologically identical 

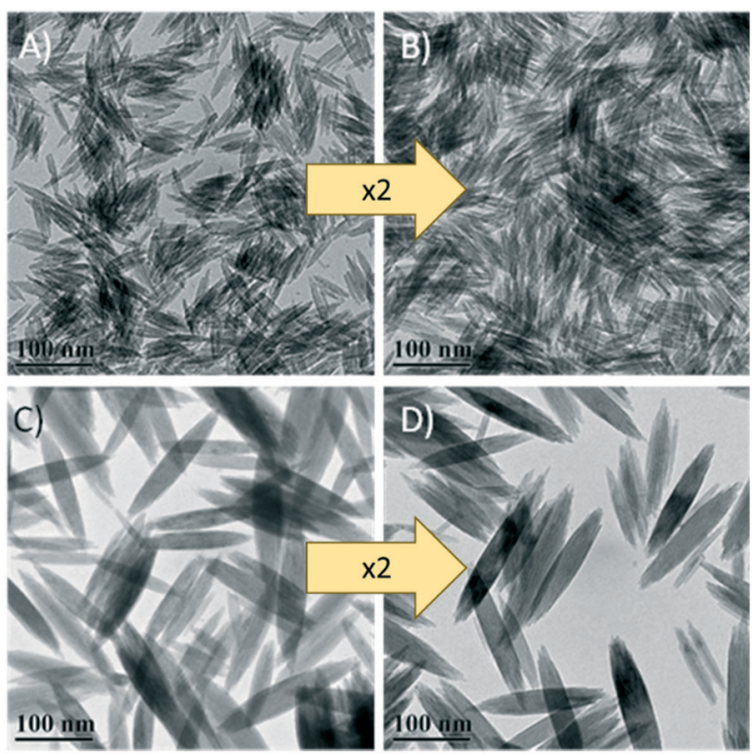

Fig. 1 TEM images of the $\beta-\mathrm{FeOOH}$ nanoellipsoids. $A$ and $C$ are the nanoellipsoids prepared with $750 \mathrm{kDa}$ and $25 \mathrm{kDa} \mathrm{PEI}$, respectively. B and $D$ are their corresponding $2 \times$ scaled-up syntheses.

nanoellipsoids. Using TGA, the yield of the initial and scaledup reactions were determined; it was found that the weight of the products was increased from $0.25 \mathrm{~g}$ to $0.76 \mathrm{~g}$ (10.5 and $16.3 \%$ yield, respectively), corresponding to a yield increase of $55 \%$ (Fig. S1 $\uparrow$ ). All further experiments were performed with the scaled-up reactions.

\section{Time-dependent growth of the nanoellipsoids}

To the best of our knowledge, the growth of $\beta$-FeOOH has not been previously investigated with respect to reaction time. Aliquots have been taken from the reaction mixture at different times and TEM images were collected. The size and aspect ratio of the nanoparticles were assessed using image analysis software ImageJ for at least 100 nanoparticles per condition. The reaction was performed in the presence of 0.2 $\mathrm{g}$ of $750 \mathrm{kDa}$ PEI and aliquots were taken between 30 and $180 \mathrm{~min}$. As can be seen from Fig. 2A, the length of the nanoellipsoids increases with time $(p$ value $=0.0005,0.04$ and 3.4 $\times 10^{-24}$ for Student $t$-test between 60 and $90 ; 90$ and $120 ; 120$ and $180 \mathrm{~min}$, respectively). The different spatial growth rate of the anisotropic structure is observed here with two times faster growth in the long axis in comparison with the short axis resulting in the same aspect ratio at all times, as shown in Fig. 2B.

Surfactants play a very important and complex role in nanoparticle synthesis in controlling facet kinetics, ${ }^{27}$ forming by-products crucial for some reactions, ${ }^{19}$ in situ generation of precursors, ${ }^{19,28,29}$ acting as reducing agents ${ }^{30}$ and even act as solvents in some cases. ${ }^{30}$ Even more complex, in the case of polymers, it is possible to crosslink particles or engulf multiple nuclei at the same time, ${ }^{31}$ which is not possible with singly functionalised molecules. To this end, the effect of PEI on the forming nanoparticles was investigated.

\section{Role of PEI on the composition and morphology of the nanoparticles}

To increase the understanding of the role of PEI in the synthesis of the material, the synthesis was performed in the presence or absence of different molecular weights of PEI. Interestingly, the $\beta$-FeOOH phase was obtained in all cases, as shown in Fig. 3. Similar to the nanoparticles prepared in the presence of PEI, the nanoparticles obtained in the absence of PEI also exhibit an elongated structure although of much less definition and homogeneity (Fig. S2†). The elongated morphology observed in the absence or presence of PEI is a result of the monoclinic crystal structure of $\beta$-FeOOH favouring anisotropic growth. ${ }^{21}$ In combination, the data suggest that PEI plays a role in determining morphology predominantly while the composition is independent of PEI.

The length of the nanoellipsoids depends on the amount of PEI used. The more PEI (25 kDa) used leads to a decrease


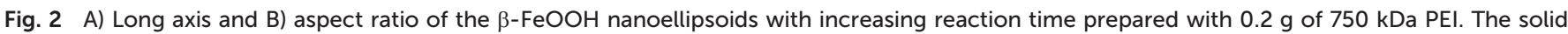
line is a guide to the eye. 


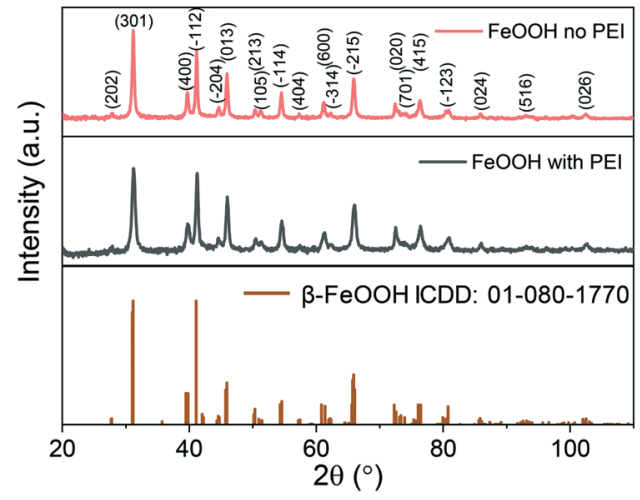

Fig. 3 XRD patterns of the nanoparticles formed in the presence or absence of PEI.

in the length of the long axis of the nanoparticles from 225 $\mathrm{nm}$ to $25 \mathrm{~nm}$, as shown in Fig. 4. The aspect ratio of the ellipsoids remains the same at a value of 6 and therefore the amount of PEI can be used to tune the size of the resulting
$\beta$-FeOOH nanoellipsoids while retaining the same aspect ratio. The homogeneity of the nanoellipsoids remains high over the PEI amounts tested $(6-300 \mathrm{mg})$. A similar trend is observed with PEI with a molecular weight of $750 \mathrm{kDa}$ for both the size and the aspect ratio of the nanoparticles with the length of the nanoellipsoids between $50-25 \mathrm{~nm}$ and a constant aspect ratio of 5 (Fig. S3†).

As $\beta-\mathrm{FeOOH}$ is paramagnetic, we studied the reduction of these nanoellipsoids with both mild and strong reducing agents under variable experimental conditions, in an attempt to form $\mathrm{Fe}_{3} \mathrm{O}_{4}$ nanoparticles.

Effects of oleylamine high-temperature reaction with $\beta$-FeOOH on the composition, morphology and magnetic properties of the nanoparticles

It was previously reported that oleylamine could reduce $\beta$-FeOOH to $\mathrm{Fe}_{3} \mathrm{O}_{4}$ as solid nanorods. ${ }^{17}$ We employed this method to convert the nanoellipsoids to $\mathrm{Fe}_{3} \mathrm{O}_{4}$ but instead porous nanoellipsoids of an $\mathrm{FeOOH}$ phase with
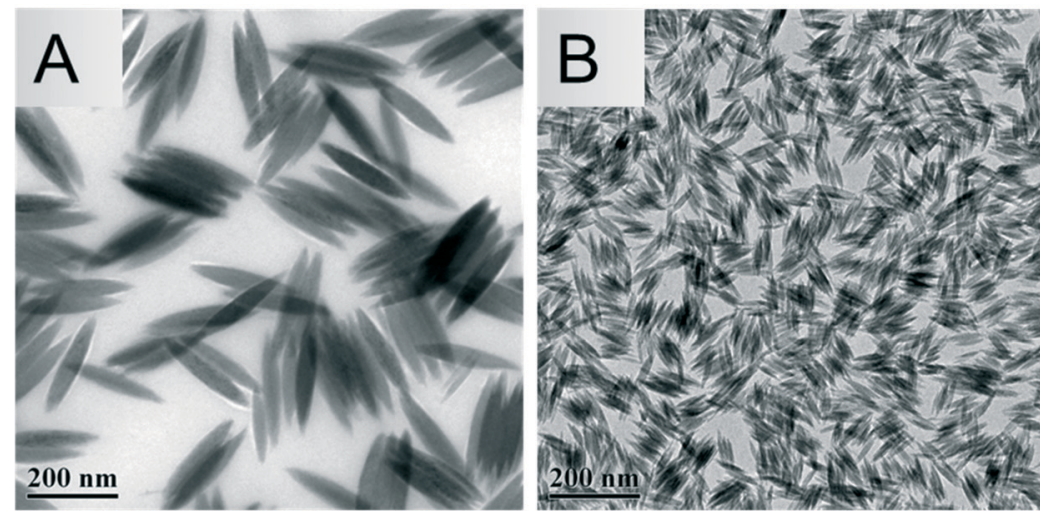

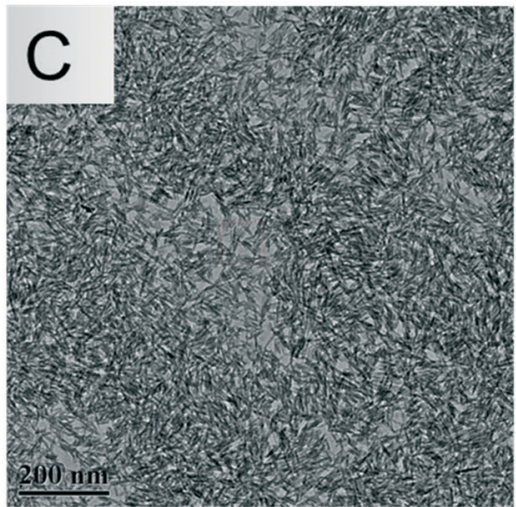

Amount of PEI

\section{Size}


Fig. $4 \mathrm{~A}-\mathrm{C}$ are the TEM images of the $\beta-\mathrm{FeOOH}$ nanoellipsoids synthesised with 10, 100 and $300 \mathrm{mg}$ of $25 \mathrm{kDa} P E \mathrm{l}$, respectively. D) Size dependence and $\mathrm{E}$, aspect ratio change with different amounts of $\mathrm{PEI}$. 
A



C

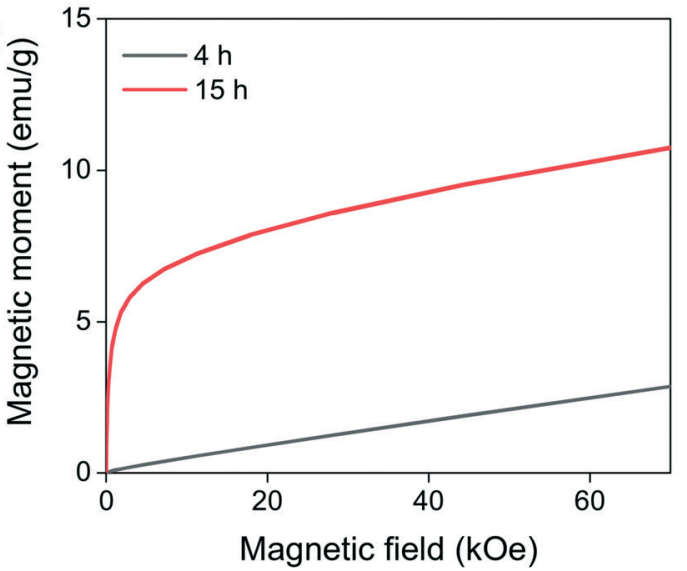

$\mathrm{B}$
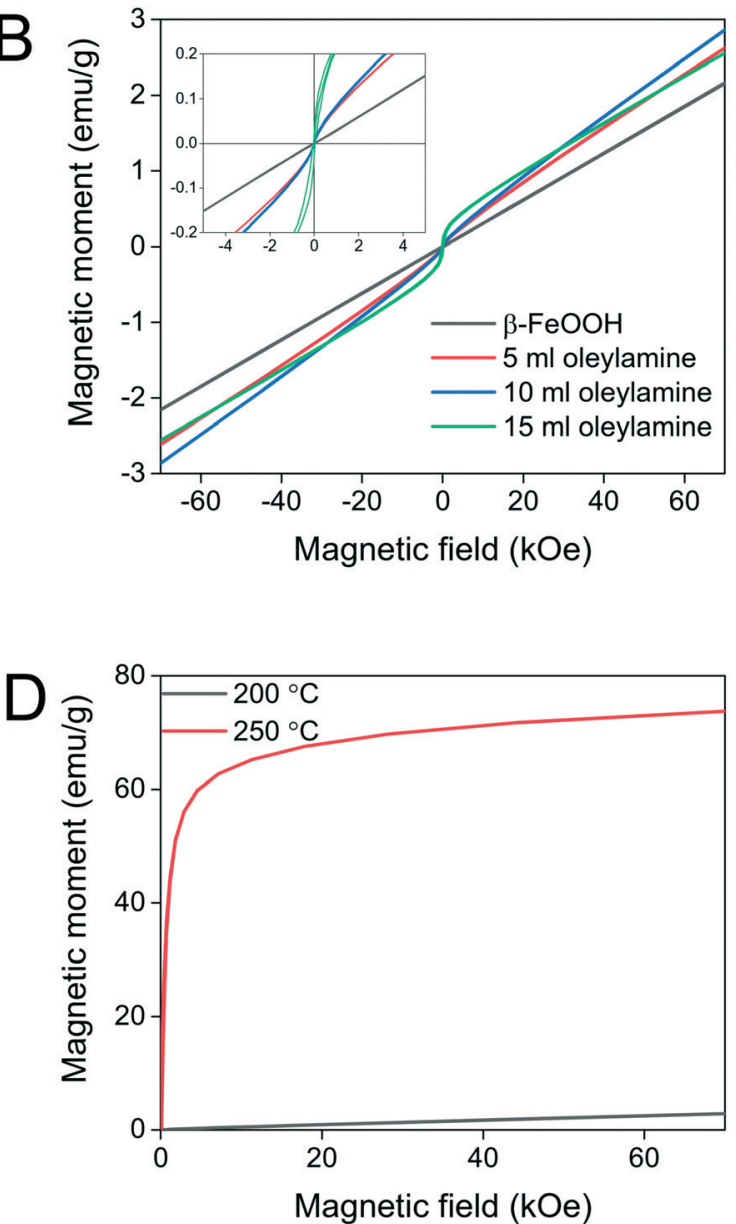

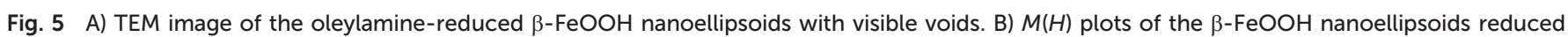


plots of the oleylamine-reduced $\beta-\mathrm{FeOOH}$ nanoellipsoids at different temperatures.

orthorhombic crystal habit were obtained, as shown in Fig. 5A and S4.† The pores could be exploited for potential application in drug delivery. The porous and hollow ellipsoids are believed to be the result of the dehydration of the oxide-hydroxide phase to form an oxide. ${ }^{26}$ Chandy et al. indicated that the chloride and water molecules from the tunnels in the $\beta-\mathrm{FeOOH}$ crystal structure are partially removed due to the thermal treatment. ${ }^{32}$ Consequently, there is a contraction in the crystal lattice changing from the monoclinic structure of $\beta$-FeOOH to orthorhombic $\mathrm{FeOOH}$ before converting to haematite $\left(\alpha-\mathrm{Fe}_{2} \mathrm{O}_{3}\right)$. This is true in this case of reacting with oleylamine at high temperature. It is also stated that further heating treatment at $320{ }^{\circ} \mathrm{C}$ of this $\mathrm{FeOOH}$ would result in $\alpha-\mathrm{Fe}_{2} \mathrm{O}_{3}$ as with all the iron oxide-hydroxide phases. ${ }^{33}$ The intensity of the peaks on the diffraction patterns obtained from XRD is low suggesting poor crystallinity, which might be due to a transitioning phase in-between the oxide-hydroxide and the oxide phases. Previously, a single condition has been used to reduce $\beta$-FeOOH to iron oxide. Herein, we investigated different experimental parameters such as the amount of oleylamine, reaction time and temperature in at- tempts to preserve the shape and control the extent of phase transformation to increase the crystallinity.

The amount of oleylamine used during the reaction did not make a difference with regards to the material formed as it can be concluded from the XRD data shown in Fig. S4. $\dagger$ Similarly, the size of the resulting nanoparticles also remained constant with changing the amount of oleylamine used (Fig. S5 $\dagger$ ). Oleylamine was found to coordinate on the surface of the nanoparticles by infrared spectroscopy (Fig. S6†).

Even though the colour of the nanoparticles changes from light brown before reaction with oleylamine to black after reaction, which is indicative of $\mathrm{Fe}_{3} \mathrm{O}_{4}$, the magnetic behaviour of the resulting particles was almost purely paramagnetic as can be seen from magnetometry measurements shown in Fig. 5B. The nanoellipsoids after reaction with oleylamine have a shorter long axis by approximately $10 \mathrm{~nm}$ while their width remained the same, essentially decreasing their aspect ratio. The reaction time seems to have a small effect on the magnetic properties of the material, as shown in Fig. 5C. From the shape of the magnetisation curve, it can be seen 
that a paramagnetic component of $\mathrm{FeOOH}$ remains in the material because a plateau is not reached even at high applied magnetic fields although an iron oxide ferrimagnetic phase is formed in a higher extent as indicated from the steep increase of magnetisation at low applied magnetic fields which is absent at shorter reaction times.

Lastly, the reaction temperature was further increased to $250{ }^{\circ} \mathrm{C}$ to overcome any thermodynamic or kinetic barriers for atom rearrangement and also promote the formation of highly crystalline materials. The temperature increase resulted in phase transformation to $\mathrm{Fe}_{3} \mathrm{O}_{4}$ to a great extent as can be concluded from the data in Fig. 5D with only a small contribution of $\mathrm{FeOOH}$, as shown from the XRD measurement (Fig. S7 $\dagger$ ). The magnetic properties increased manifold to values typical of high-quality magnetic nanoparticles. Unfortunately, the morphology of the resulting nanoparticles is lost, and a variety of shapes are formed including cuboid and parallelepiped shapes (Fig. S8†).

Other high-temperature reductions observed in the literature often result in sintering, merging many nanoparticles together and losing the nanosize and morphological characteristics. To promote reduction and allow the reaction to proceed at lower temperature, hydrazine has been used as a stronger, water-miscible reducing agent.

\section{Physicochemical changes of $\beta$-FeOOH induced by hydrazine reduction}

As far as we know, hydrazine has not been previously used to reduce the $\beta$-FeOOH nanoellipsoids. Hydrazine, a stronger reducing agent than oleylamine, was employed to induce the reduction at lower temperature to prevent thermal dehydroxylation and possibly avoid porous/hollow structure formation. The transformation was shown to follow a dissolution-recrystallization mechanism through the formation of $\mathrm{Fe}(\mathrm{OH})_{4}{ }^{-}$species in a basic medium. ${ }^{34} \mathrm{~A} \mathrm{pH}$ value of 10.5 was shown to induce the slowest rate of transformation within the window of 9-11.5 where this process takes place. $^{33}$ By adopting the $\mathrm{pH}$ value of 10.5 , we hypothesised that the transformation could be controlled due to its slow kinetics possibly retaining the overall shape of the nanoparticles. The reduction reaction of $\beta-\mathrm{FeOOH}$ with hydrazine is described in eqn (1).

$$
12 \beta-\mathrm{FeOOH}+\mathrm{N}_{2} \mathrm{H}_{4} \rightarrow 4 \mathrm{Fe}_{3} \mathrm{O}_{4}+8 \mathrm{H}_{2} \mathrm{O}+\mathrm{N}_{2}
$$

The reduction with hydrazine is fast and occurs at $100{ }^{\circ} \mathrm{C}$ as opposed to $250{ }^{\circ} \mathrm{C}$ with oleylamine. The reaction mixture turns from brown to black after approximately $10 \mathrm{~min}$ of stirring and aliquots from 30-240 min show the same morphology at every time-point indicating that the reaction is complete after $30 \mathrm{~min}$ (Fig. S9†). The XRD patterns reveal primarily an iron oxide phase with a few low intensity peaks indexed to $\alpha-\mathrm{FeOOH}$, as shown in Fig. 6 .

The resulting nanoparticles from hydrazine reduction lose their ellipsoidal morphology producing faceted nanoparticles

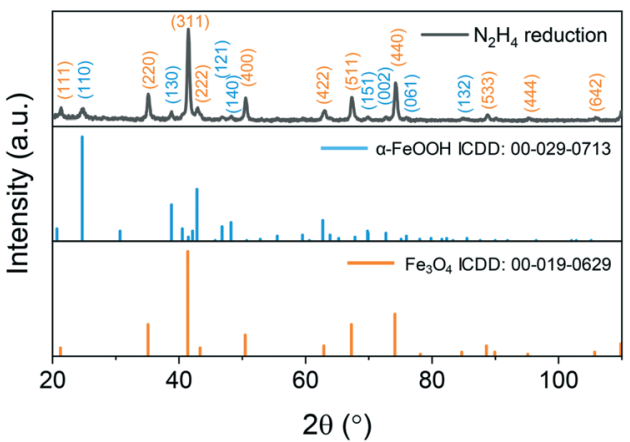

Fig. 6 XRD patterns of the hydrazine-reduced $\beta-\mathrm{FeOOH}$ nanoellipsoids at $100{ }^{\circ} \mathrm{C}$ after $4 \mathrm{~h}$ of reaction. Indexed reflections are colour-coded with respect to the reference patterns.

including cuboids, wires and truncated triangles (Fig. S7†). Due to the loss of their morphology, PEI was introduced into the reaction mixture to reduce the rate of reaction by coordinating on the surface of $\beta$-FeOOH and retain the shape by coordinating on the surface of the iron oxide limiting further growth. Initially, $0.2 \mathrm{~g}$ of PEI was introduced, and the reaction time was varied from $30 \mathrm{~min}$ to $4 \mathrm{~h}$. At $30 \mathrm{~min}$, hollow nanoellipsoids prevail in the TEM image with a few wires also being visibly shown in Fig. 7A. The TEM image of the prolonged reaction time shows mostly the nanowires with some rod-shaped particles as well shown in Fig. 7B. The XRD data show a mixture of $\mathrm{Fe}_{3} \mathrm{O}_{4}$ and $\alpha-\mathrm{FeOOH}$, as shown in Fig. 7C. When the reaction time was increased to $4 \mathrm{~h}$, the product was an almost pure $\alpha-\mathrm{FeOOH}$ phase with some low intensity $\mathrm{Fe}_{3} \mathrm{O}_{4}$ peaks (for clarity in Fig. 7C, not all reflections have been indexed). The formation of $\alpha-\mathrm{FeOOH}$ with increasing reaction time and disappearing of $\mathrm{Fe}_{3} \mathrm{O}_{4}$ can be monitored by the 220, 311 and 400 reflections of $\mathrm{Fe}_{3} \mathrm{O}_{4}$ at $35.1^{\circ}$,

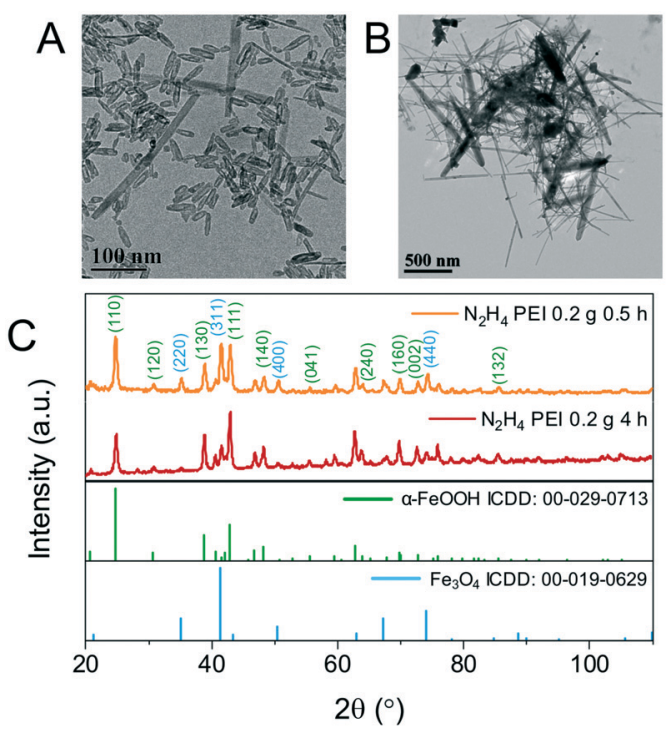

Fig. $7 \mathrm{~A}$ and $\mathrm{B}$ are the TEM images of $\beta-\mathrm{FeOOH}$ reduced in the presence of $\mathrm{N}_{2} \mathrm{H}_{4}$ and $0.2 \mathrm{~g} \mathrm{PEI}$ at $100{ }^{\circ} \mathrm{C}$ for $0.5 \mathrm{~h}$ and $4 \mathrm{~h}$, respectively. $\mathrm{C}$ is the XRD patterns of the nanoparticles in $\mathrm{A}$ and $\mathrm{B}$. Indexed reflections are colour-coded with respect to the reference patterns. 

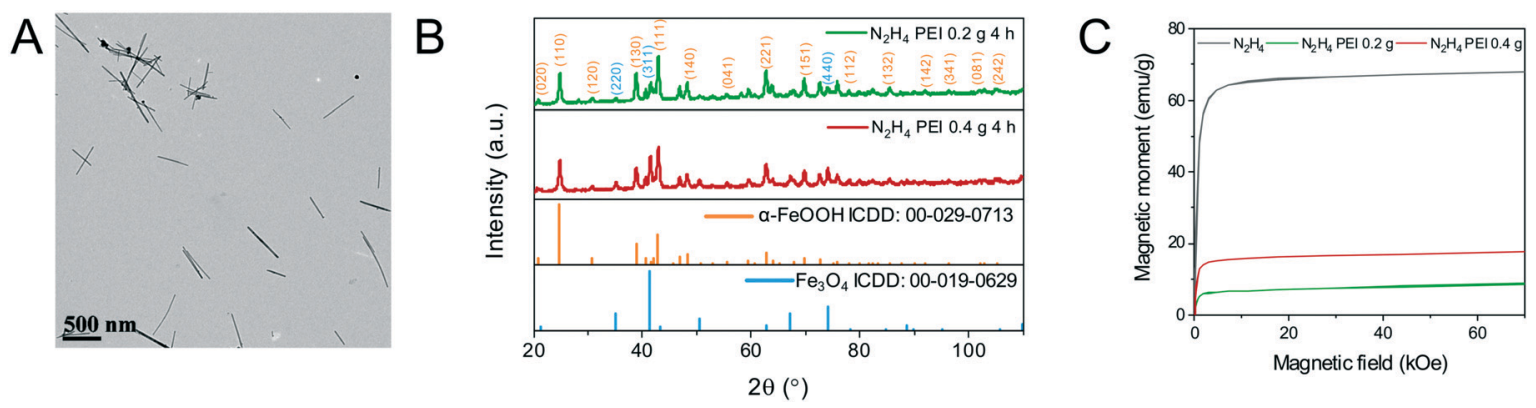

Fig. 8 A) TEM image of the $\beta$ - $\mathrm{FeOOH}$ nanoellipsoids reduced by $\mathrm{N}_{2} \mathrm{H}_{4}$ at $100{ }^{\circ} \mathrm{C}$ in the presence of $0.4 \mathrm{~g}$ PEI. B) XRD patterns of the $\beta$-FeOOH nanoellipsoids reduced by $\mathrm{N}_{2} \mathrm{H}_{4}$ at $100{ }^{\circ} \mathrm{C}$ in the presence of 0.2 and $0.4 \mathrm{~g} \mathrm{PEl}$. C) $M(H)$ plots of the $\beta$-FeOOH nanoellipsoids reduced by $\mathrm{N}_{2} \mathrm{H}_{4}$ in the absence or presence and different amounts of PEI.

$41.3^{\circ}$ and $50.4^{\circ}$, respectively. From the population of the wires on the TEM images and in accordance with the XRD data, it can be concluded that the nanowires are composed of $\alpha-\mathrm{FeOOH}$ and a longer reaction time favours the formation of $\alpha$-FeOOH making this polymorphic transformation rather than reduction. The effect arises from the presence of PEI only under the same experimental conditions but in its absence, only $\mathrm{Fe}_{3} \mathrm{O}_{4}$ is obtained. This might be the result of strong coordination of the amine groups of PEI with the iron atoms which might prohibit or considerably slow their desorption from the nanoellipsoids to perform the dissolution-recrystallization reduction mechanism and favour polymorphic transformation instead. To further understand what the role of PEI is in these reactions, its amount was doubled to $0.4 \mathrm{~g}$ and the reaction was carried out for $4 \mathrm{~h}$. The composition of the resulting materials is similar to the $0.2 \mathrm{~g}$ PEI reaction, a predominant $\alpha$-FeOOH with some $\mathrm{Fe}_{3} \mathrm{O}_{4}$ peaks although the TEM image shows smaller nanowires as shown in Fig. 8A and B. The products of reduction by hydrazine in the presence and absence of PEI were analysed by SQUID-VSM. The results shown in Fig. 8C show high saturation magnetisation for the nanoparticles reduced in the presence of hydrazine only while when PEI was used, the saturation magnetisation was significantly lower which agrees with the XRD data of a dominant $\alpha-\mathrm{FeOOH}$ phase. These data suggest that the formation of $\alpha-\mathrm{FeOOH}$ is favoured in the presence of PEI while the more magnetic phase $\mathrm{Fe}_{3} \mathrm{O}_{4}$ is formed in its absence.

\section{Conclusions}

These advances in controlling the size of $\beta$-FeOOH nanoparticles will find use in oxide-hydroxide based catalysis, environmental remediation and possibly in anisotropic magnetic nanoparticle synthesis, all of which have been previously attempted using $\beta$-FeOOH-based materials.

A high degree of control over the synthesis of the $\beta-\mathrm{FeOOH}$ nanoellipsoids has been achieved by changing the molecular weight and amount of PEI used in the reaction providing a wider range of sizes to scientists interested in iron oxide-hydroxide based nanomaterials. Experimental variables in the synthesis of the $\beta$-FeOOH nanoellipsoids were studied and the PEI used during the preparation of the material was shown to play a morphological role only. The long-axis growth of the nanoellipsoids is time-dependent and also affected by the molecular weight of the PEI used. The aspect ratio of the nanoellipsoids is independent of the reaction time but it can also be changed by changing the molecular weight of PEI.

Oleylamine used as a mild reducing agent to convert the $\beta$-FeOOH nanoellipsoids to porous iron oxide nanoellipsoids at all reaction times and amounts tested at $200{ }^{\circ} \mathrm{C}$ but magnetic measurements and XRD did not show a ferrite material. Increasing the temperature to $250{ }^{\circ} \mathrm{C}$ drives the transformation to completion according to XRD and magnetometry studies which is accompanied by a loss of morphology.

Hydrazine employed as a strong reducing agent also caused a loss of morphology when used alone but successfully forms $\mathrm{Fe}_{3} \mathrm{O}_{4}$ confirmed by XRD and magnetometry fast at lower temperature. When PEI is present during reduction with hydrazine, the morphology is retained, hollow nanoparticles are observed along with a mixture of $\mathrm{Fe}_{3} \mathrm{O}_{4}$ and $\alpha$-FeOOH whose ratio is dependent on the amount of PEI present in the reaction. From the experiments performed herein, it is shown that when phase transformation occurs, a loss of morphology is also observed while when the morphology is preserved, polymorphic transformation takes place instead of reduction indicating the need for further studies to achieve the formation of anisotropic iron oxide nanoparticles by using $\mathrm{FeOOH}$ materials as precursors.

\section{Conflicts of interest}

The authors declare no conflicts of interest.

\section{Acknowledgements}

G. Kasparis would like to thank EPSRC for his PhD studentship. J. M. Tuffnell gratefully acknowledges financial support from the Institute for the Physics of Living Systems. N. T. K. Thanh thanks EPSRC (EP/M018016/1 and EP/M015157/1) and AOARD (FA2386-17-1-4042 award) for funding.

\section{Notes and references}

1 A. Meffre, B. Mehdaoui and V. Connord, et al., Complex nano-objects displaying both magnetic and catalytic 
properties: A proof of concept for magnetically induced heterogeneous catalysis, Nano Lett., 2015, 15, 3241-3248, DOI: 10.1021/acs.nanolett.5b00446.

2 M. E. Khan, M. M. Khan and M. H. Cho, Recent progress of metal-graphene nanostructures in photocatalysis, Nanoscale, 2018, 10(20), 9427-9440, DOI: 10.1039/c8nr03500h.

3 J. Wang, Y. Li and L. Deng, et al., High-Performance Photothermal Conversion of Narrow-Bandgap $\mathrm{Ti}_{2} \mathrm{O}_{3}$ Nanoparticles, Adv. Mater., 2016, 1-6, DOI: 10.1002/ adma.201603730.

4 S. Ghosh and R. N. Basu, Multifunctional nanostructured electrocatalysts for energy conversion and storage: current status and perspectives, Nanoscale, 2018, 10(24), 11241-11280, DOI: 10.1039/c8nr01032c.

5 D. Karponis, M. Azzawi and A. Seifalian, An arsenal of magnetic nanoparticles; perspectives in the treatment of cancer, Nanomedicine, 2016, 11(16), 2215-2232, DOI: 10.2217/nnm-2016-0113.

6 Q. A. Pankhurst, N. K. T. Thanh, S. K. Jones and J. Dobson, Progress in applications of magnetic nanoparticles in biomedicine, J. Phys. D: Appl. Phys., 2009, 42, 224001.

7 N. T. K. Thanh, Clinical Applications of Magnetic Nanoparticle, CRC press, London, 2017.

8 J. Zhu, S. Wei and M. Chen, et al., Magnetic nanocomposites for environmental remediation, Adv. Powder Technol., 2013, 24(2), 459-467, DOI: 10.1016/j.apt.2012.10.012.

9 F. Li, D. MacFarlane and J. Zhang, Recent Advances in Nanoengineering of Electrocatalysts for $\mathrm{CO} 2$ Reduction, Nanoscale, 2018, 6235-6260, DOI: 10.1039/C7NR09620H.

10 J. P. Zimmer, X. Liu and Y. Yang, et al., Synthesis of nonstoichiometric zinc ferrite nanoparticles with extraordinary room temperature magnetism and their diverse applications, J. Mater. Chem. C, 2013, 1, 2875-2885, DOI: 10.1039/c3tc00790a.

11 H. Chen, L. Shao, Q. Li and J. Wang, Gold nanorods and their plasmonic properties, Chem. Soc. Rev., 2013, 42(7), 2679-2724, DOI: 10.1039/c2cs35367a.

12 X. Wang, Z. Li, J. Shi and Y. Yu, One-dimensional titanium dioxide nanomaterials: Nanowires, nanorods, and nanobelts, Chem. Rev., 2014, 114(19), 9346-9384, DOI: 10.1021/cr400633s.

13 P. K. Jain, K. S. Lee, I. El-Sayed and M. El-Sayed, Calculated Absorption and Scattering Properties of Gold Nanoparticles of Different Size, Shape, and Composition: Applications in Biological Imaging and Biomedicine, J. Phys. Chem. B, 2006, 110, 7238-7248, DOI: 10.1021/jp057170o.

14 Y. A. N. Geng, P. Dalhaimer, S. Cai, R. Tsai, T. Minko and D. E. Discher, Shape effects of filaments versus spherical particles in flow and drug delivery, Nat. Nanotechnol., 2007, 2(4), 249-255, DOI: 10.1038/nnano.2007.70.Shape.

15 P. Kolhar, A. C. Anselmo and V. Gupta, et al., Using shape effects to target antibody-coated nanoparticles to lung and brain endothelium, Proc. Natl. Acad. Sci. U. S. A., 2013, 110(26), 10753-10758, DOI: 10.1073/pnas.1308345110.

16 B. J. Wiley, Y. Chen and J. McLellan, et al., Synthesis and Optical Properties of Silver Nanobars and Nanorice, Nano Lett., 2007, 7(Copyright (C) 2015 American Chemical Society
(ACS). All Rights Reserved), 1032-1036, DOI: 10.1021/ nl070214f.

17 J. Mohapatra, A. Mitra, H. Tyagi, D. Bahadur and M. Aslam, Iron oxide nanorods as high-performance magnetic resonance imaging contrast agents, Nanoscale, 2015, 7(20), 9174-9184, DOI: 10.1039/c5nr00055f.

18 R. Das, J. Alonso and P. Z. Nemati, et al., Tunable High Aspect Ratio Iron Oxide Nanorods for Enhanced Hyperthermia, J. Phys. Chem. C, 2016, 120(18), 10086-10093, DOI: 10.1021/acs.jpcc.6b02006.

19 H. Sun, B. Chen, X. Jiao, Z. Jiang, Z. Qin and D. Chen, Solvothermal synthesis of tunable electroactive magnetite nanorods by controlling the side reaction, J. Phys. Chem. C, 2012, 116(9), 5476-5481, DOI: 10.1021/jp211986a.

20 Y. Wang and H. Yang, Synthesis of iron oxide nanorods and nanocubes in an imidazolium ionic liquid, Chem. Eng. J., 2009, 147(1), 71-78, DOI: 10.1016/j.cej.2008.11.043.

21 J. Zhao, W. Lin, Q. Chang, W. Li and Y. Lai, Adsorptive characteristics of akaganeite and its environmental applications: a review, Environ. Technol. Rev., 2012, 2515, 1-13, DOI: 10.1080/09593330.2012.701239.

22 C. Blanco-Andujar, D. Ortega, Q. A. Pankhurst and N. T. K. Thanh, Elucidating the morphological and structural evolution of iron oxide nanoparticles formed by sodium carbonate in aqueous medium, J. Mater. Chem., 2012, 22(25), 12498, DOI: 10.1039/c2jm31295f.

23 Mozo S. Lentijo, E. Zuddas, A. Casu and A. Falqui, Synthesizing Iron Oxide Nanostructures: The Polyethylenenemine (PEI) Role, Crystals, 2017, 7(1), 22, DOI: 10.3390/cryst7010022.

24 C. Lui, X. Wu, T. Klemmer, N. Shukla and D. Weller, Reduction of sintering during annealing of ePt nanoparticles coated with iron oxide, Chem. Mater., 2005, 17(6), 620-625.

25 Y. Piao, J. Kim and H. B. Na, et al., Wrap-bake-peel process for nanostructural transformation from beta-FeOOH nanorods to biocompatible iron oxide nanocapsules, Nat. Mater., 2008, 7(3), 242-247, DOI: 10.1038/nmat2118.

26 W. Xu, M. Wang and Z. Li, et al., Chemical Transformation of Colloidal Nanostructures with Morphological Preservation by Surface-Protection with Capping Ligands, Nano Lett., 2017, 17(4), 2713-2718.

27 Y. Xia, Y. Xiong, B. Lim and S. E. Skrabalak, Shapecontrolled synthesis of metal nanocrystals: Simple chemistry meets complex physics?, Angew. Chem., Int. Ed., 2009, 48(1), 60-103, DOI: 10.1002/anie.200802248.

28 N. T. K. Thanh, N. T. Dung and L. D. Tung, et al., Synthesis of magnetic cobalt ferrite nanoparticles with controlled morphology, monodispersity and composition: the influence of solvent, surfactant, reductant and synthetic condition, Nanoscale, 2015, 7, 19596-19610.

29 S. N. Rishikeshi, S. S. Joshi, M. K. Temgire and J. R. Bellare, Chain length dependence of polyol synthesis of zinc ferrite nanoparticles: why is diethylene glycol so different?, Dalton Trans., 2013, 42(15), 5430-5438, DOI: 10.1039/c2dt32026f.

30 S. Mourdikoudis and L. M. Liz-Marzán, Oleylamine in nanoparticle synthesis, Chem. Mater., 2013, 25(9), 1465-1476, DOI: $10.1021 / \mathrm{cm} 4000476$. 
31 R. Sondjaja, H. T. Alan and M. K. C. Tam, Clustering of magnetic nanoparticles using a double hydrophilic block copolymer, poly(ethylene oxide)-b-poly(acrylic acid), J. Magn. Magn. Mater., 2009, 321(16), 2393-2397, DOI: 10.1016/j. jmmm.2009.02.136.

32 K. Chandy, Thermal transformation of b-ferric oxyhydroxide, Mineral. Mag., 1965, 35(272), 666-669.
33 R. M. Cornell and U. Schwertmann, The Iron Oxides: Structure, Properties, Reactions, Occurences and Uses, WileyVCH, Weinheim, 2004, DOI: 10.1002/3527602097.ch1.

34 M. A. Blesa, M. Mijalchik, M. Villegas and G. Rigotti, Transformation of akaganeite into magnetite in aqueous hydrazine suspensions, React. Solids, 1986, 2(1-2), 85-94, DOI: 10.1016/0168-7336(86)80066-3. 